\title{
Treatment of Relapsed Acute Myeloid Leukemia
}

\section{Felicitas Thol, MD \\ Arnold Ganser, MD*}

\author{
Address \\ *Department of Hematology, Hemostasis, Oncology and Stem Cell Transplanta- \\ tion, Hannover Medical School, Carl-Neuberg-Str. 1, 30625, Hannover, Germany \\ Email: ganser.arnold@mh-hannover.de
}

Published online: 29 June 2020

(C) The Author(s) 2020

This article is part of the Topical Collection on Leukemia

Keywords Relapse AML · Salavage chemotherapy · Venetoclax · FLT3 inhibitors · IDH1/IDH2 inhibitors · CPX-351

\section{Opinion statement}

Relapse is still a common scenario in acute myeloid leukemia (AML) treatment and occurs in $40-50 \%$ of younger and the great majority of elderly patients. The prognosis in relapsed AML patients is generally poor but depends largely on the timing of relapse (early versus late) and the possibility of allogeneic hematopoietic stem cell transplantation (HSCT). At the time of relapse, we again perform a mutational screening and cytogenetic analysis in all AML patients as clonal evolution of disease is frequent. Clinical trials should be first priority in all relapsed patients. In fit patients without prior transplant, we aim to perform HSCT after salvage therapy. In AML patients relapsing after HSCT and good performance status, intensive therapy can be considered with subsequent cellular therapy such as donor lymphocyte infusion (DLI) or a second HSCT. However, less than $20 \%$ of these patients are alive after 5 years. For those patients that are unfit, the therapeutic aim is to prolong life with acceptable quality of life. Here, hypomethylating agents (HMA), low-dose AraC (LDAC), and solely cytoreductive therapy with hydroxurea are options depending on first-line therapy. For those patients that have not been treated with venetoclax in first line, the combination therapy of venetoclax with demethylating agents achieves encouraging response rates. Venetoclax is currently also studied in combination with intensive salvage therapy. Importantly, for patients with isocitrate dehydrogenase (IDH) 1/2-mutated AML, ivosidenib, an IDH1 inhibitor, and enasidenib, an IDH2 inhibitor, present well-tolerated options in the setting of refractory or relapsed $(r / r)$ disease even in elderly and heavily pretreated patients with response rates of $30-40 \%$. Both substances have been approved by the U.S. Food and Drug Administration (FDA) for r/r AML patients with IDH1/2 mutations (but not yet by the European Medicines Agency (EMA)). For patients with FMS-like tyrosine kinase 3 (FLT3) mutations, treatment with the selective FLT3 inhibitor gilteritinib is well tolerated and leads to improved outcome compared with standard salvage therapy. The approval has been granted by the FDA and the EMA. Generally, we would recommend targeted therapy for IDH1/2- and FLT3-mutated AML if available. In order to improve outcome in relapsed AML, it 
will be important to intelligently combine novel substances with each other as well as chemotherapy in prospective clinical trials. The development of therapies with bispecific antibodies or chimeric antigen receptor T cells (CAR-T) are still in early development.

\section{Introduction}

Despite improvements in acute myeloid leukemia (AML) therapy, relapse is still the most challenging aspect in AML. While $10-40 \%$ of younger AML patients are primarily refractory to AML induction therapy, the number is considerably higher for patients above 60 years (40$60 \%)$ [1]. The great majority of fit patients will undergo hematopoietic stem cell transplantation (HSCT) after achieving a complete remission (CR). However, $40 \%$ of these patients relapse after HSCT [2]. Thus, refractory or relapsed $(r / r)$ AML is a very common scenario in AML. At the time of relapse, a thorough diagnostic work-up is essential. Besides cytology and morphology, we would also recommend performing a cytogenetic analysis as well as mutational screening in all relapsed patients. The need for this analysis at time of relapse is based on the high likelihood of clonal evolution of AML. With the advancement of sequencing technologies, we have learned that the mutational pattern at the time of relapse is frequently discrepant from that at diagnosis. Mutations are frequently gained or lost during the course of the disease. Furthermore, the variant allelic frequency (VAF) of gene mutations is likely to change in the course of the disease, e.g., with smaller clones becoming more dominant as well as dominant clones decreasing in size. The cytogenetic and molecular results help us to further risk stratify patients in relapse. Furthermore, some mutations like FLT3, IDH1, and IDH2 offer novel therapeutic options. Due to next-generation sequencing (NGS) being widely available, mutational screening is frequently done in a targeted panel approach covering the most frequently mutated genes in AML. If NGS is not available, we would at least recommend sequencing the "targetable" mutations (e.g., FLT3, IDH1, IDH2) as well as other prognostic mutations (e.g., NPM1, double mutated CEBPA, TP53,

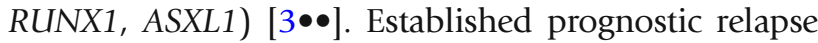
scores have integrated molecular markers in the scoring systems such as FLT3-ITD in the GOELAMS score [4]. We recommend human leukocyte antigen (HLA) typing for all patients who are fit for allogeneic stem cell transplantation at the time of diagnosis. However, if this has not been done at that time point, HLA typing and donor search should be initiated as early as possible when relapse occurs. Due to toxicities occurring in the course of the disease and during prior treatment, a through clinical evaluation of patients at the time of relapse is as important as it is at the time of diagnosis and may lead the direction for treatment planning and HSCT. Important questions at the time of relapse include whether the patient can be intensively treated with the aim of subsequent HSCT or other cellular therapies and how likely it is that the patient responds to available relapse therapies.

\section{Treatment}

Standard treatment for relapse in fit patients with or without prior allogeneic stem cell transplantation

AML patients relapsing after chemotherapy still have a curative treatment approach if they are fit enough to undergo HSCT [5]. Salvage therapy has the aim to induce a cytological remission before HSCT and serves as the "bridge to transplantation". Clinical trials are our first choice for all eligible patients. There is no standard regimen for salvage therapy and different regimens have shown similar results [5]. However, important components of salvage therapy include an anthracyclin and high-dose cytarabine. Commonly, a purine analog (e.g., fludarabine or cladribine) or etoposide, a topoisomerase II inhibitor, is added 
to this regimen [5]. A widely used regimen is the FLAG-IDA protocol that includes fludarabine, high-dose cytarabine, idarubicin, and granulocyte colonystimulating factor (G-CSF). With this regimen, $50 \%$ of $\mathrm{r} / \mathrm{r}$ patients achieve a CR [6]. The FLAG-IDA regimen also presents a frequently used control arm for newer drugs or regimens in clinical trials for $\mathrm{r} / \mathrm{r}$ AML. The salvage therapyinduced remission is usually only shortly sustained and performing HSCT in a timely manner is critical. For patients relapsing after HSCT, the outlook is even more dismal [7]. An analysis of the Center for International Blood and Marrow Transplant Research (CIBMTR) showed that of 1788 AML patients relapsing after allografts, only $23 \%$ of patients were alive at 1 year after relapse [8]. Patients relapsing early after HSCT ( $<6$ months) have a significantly poorer outcome compared with patients with a late relapse [7, 8]. Especially in the latter group, salvage therapy followed by a repeated HSCT or other cellular therapies such as DLIs can be discussed. There is currently no evidence that a change of donor for second HSCT is of advantage. A European Society for Blood and Marrow Transplantation (EBMT)-based registry study of 418 AML patients relapsing after first HSCT compared outcome of patients receiving a second HSCT compared with DLI [9]. Overall survival (OS) between the two groups was not significantly different with the 2-year OS being $26 \%$ and $25 \%$ and the 5 -year OS being $19 \%$ versus $15 \%$ respectively [9]. Importantly, this study underscored that outcome of patients with early relapse after HSCT is dismal with 9-11\% 2-year OS and 4-9\% 5-year OS [9]. Because of these poor results, novel therapies are urgently needed. For specific molecular groups (FLT3- or IDH1/2-mutated patients), treatment with targeted therapies need to be integrated into therapeutic decision making (see below).

\section{Relapse in unfit patients after non-intensive therapy}

Nearly all elderly patients become refractory or relapse after non-intensive therapy. While HMA treatment such as azacitidine has improved clinical outcome of this patient population, only few patients can be cured with these regimens [10]. While two new substances (venetoclax with HMA or LDAC, glasdegib with LDAC) have been recently approved by the FDA for newly diagnosed elderly AML patients, we still have to learn about the long-term results that can be achieved with these novel combination therapies. Whenever possible, also unfit $\mathrm{r} / \mathrm{r}$ AML patients should be included in a clinical trial. Approved therapeutic options are very limited in this situation so that cytoreduction with hydroxyurea or LDAC and symptomatic therapy with transfusions and anti-infective therapy might be the only possible therapeutic measures.

\section{Novel substances-non-targeted}

\section{Venetoclax}

Venetoclax is a highly selective, oral small-molecule B cell leukemia/lymphoma-2 (BCL2) inhibitor. Members of the BCL protein family regulate apoptosis in a proor anti-apoptotic manner. BCL2 shows anti-apoptotic activity, is highly expressed in leukemia stem cells, and maintains myeloblast survival $[11,12]$. Thus, it is not surprising that BCL2 inhibition is a promising target in AML. In a single-arm phase II study, 32 patients with high-risk r/r AML or unfit for intensive chemotherapy received $800 \mathrm{mg}$ venetoclax monotherapy. In this study, monotherapy 
achieved response rates according to International Working Group (IWG) criteria in $19 \%$ of patients while additional $19 \%$ of patients showed some antileukemic response that did not fulfill IWG criteria [13]. Similarly, in a very small case serious of seven patients with secondary AML (sAML) who were not eligible for intensive chemotherapy and were refractory to HMA treatment, 2 patients achieved a CR with venetoclax monotherapy that was durable with a PFS of 505 days and 352 days [14]. Both studies indicate that venetoclax monotherapy shows some activity in the $\mathrm{r} / \mathrm{r}$ AML setting. Nevertheless, these response rates are still unsatisfactory in AML as compared with the impressive results in chronic lymphocytic leukemia. Therefore, the combination therapy of venetoclax is more appealing. Very promising data in first-line treatment of elderly AML patients treated with HMA and venetoclax combination have been reported [15]. In this study, a CR and with incomplete hematological recovery (CRi) was obtained in $67 \%$ of patients (91\% in NPM1-mutated patients). Importantly, favorable remission rates were also observed in prognostically unfavorable subgroups (highrisk cytogenetics, sAML, and TP53 mutations). The combination of LDAC and venetoclax achieved CR/CRi in 54\% of elderly previously untreated AML patients [16]. Here, lower CR/CRi rates were seen in patients with TP53 or FLT3 mutations (30\% and $44 \%$ respectively). These encouraging results led to the approval of venetoclax by the FDA in November 2018 in combination with azacitidine or decitabine or LDAC for the treatment of newly diagnosed AML in adults who are age 75 years or older, or who have comorbidities that preclude use of intensive induction chemotherapy (no approval by EMA yet). Thus, the question comes up how venetoclax combination therapy works in $\mathrm{r} / \mathrm{r}$ AML patients. In a multicenter historical study, 23 AML patients who were refractory to HMA treatment or relapsed after HMA therapy were treated with a combination of venetoclax and HMA. Forty-three percent achieved a CR or CRi in this cohort while OS was 74\% at 6 months [17]. Similar results were seen in $33 \mathrm{r} / \mathrm{r}$ AML patients treated with HMA plus venetoclax outside a clinical trial. The overall response rate here was $64 \%$ [18]. Thus, combination therapy consisting of HMA and venetoclax is promising in patients with $\mathrm{r} / \mathrm{r}$ AML. The combination therapy of venetoclax with intensive therapy is currently under investigation. In our observational study, we treated $13 \mathrm{r} / \mathrm{r}$ AML patients with FLAG-IDA in combination with venetoclax (FLAV-IDA; venetoclax given on days 1-7) and compared the results retrospectively with $81 \mathrm{r} / \mathrm{r}$ AML patients treated with FLAG-IDA alone [19]. Overall, the venetoclax combination therapy was well tolerated with no excess hematological toxicity. The ORR rate was $69 \%$ (FLA-V-IDA) versus $47 \%$ (FLAG-IDA). In a similar phase $1 \mathrm{~B}$ study, $\mathrm{r} / \mathrm{r}$ AML patients received FLAG-IDA with either venetoclax $200 \mathrm{mg}$ on days $1-21$ or subsequently on days $1-14$ due to observed infectious complications. For those patients not proceeding to HSCT, venetoclax monotherapy was given as maintenance therapy. Out of 11 evaluable patients, 8 patients (73\%) achieved a CR or CRi [20]. Both studies are early but promising. Larger prospective and randomized trials are urgently required to evaluate venetoclax in combination with intensive chemotherapy for fit $\mathrm{r} / \mathrm{r}$ AML patients. In summary, venetoclax especially in combination therapy is promising in $\mathrm{r} / \mathrm{r} \mathrm{AML}$. 
plasma concentrations as well as enrichment of the substances in the bone marrow. CPX-351 was first studied in $\mathrm{r} / \mathrm{r}$ AML patients and high-risk MDS patients in a dose-finding trial. Of $43 \mathrm{r} / \mathrm{r}$ AML patients, 9 patients showed a CR and 1 patient a CRi [21]. In a subsequent randomized phase II trial, CPX-351 was compared with standard salvage chemotherapy in $\mathrm{r} / \mathrm{r}$ AML patients [22]. In the whole cohort, there was no statistically significant 1 -year survival improvement observed. However, in the subgroup of patients with poor risk features patients, the CPX-351 arm showed higher response rates (CR and CRi) (39.3\% versus $27.6 \%)$ with a statistically significant prolongation of OS (6.6\% versus $4.2 \%)$ and EFS (2 versus 1.2 months). The observed benefit in $\mathrm{r} / \mathrm{r}$ AML patients with poor risk features led to a randomized phase III trial in elderly patients with newly diagnosed high-risk AML that included therapy-related AML (t-AML), sAML after MDS or chronic myelomonocytic leukemia (CMML), or de novo AML with MDS-related cytogenetic changes comparing CPX-351 with standard induction therapy [23]. Here, CPX-351 significantly increased median OS (9.56 versus 5.95 months) and overall remission rates (CR and CRi) (47.7\% versus 33.3\%). The toxicity profile was similar to that seen with standard chemotherapy with the exception of prolonged neutropenia and thrombocytopenia in the investigational arm. These trial results resulted in CPX-351 being approved by the FDA in 2017 and the EMA in 2018 for the treatment of adults with newly diagnosed therapy-related acute myeloid leukemia (t-AML) or acute myeloid leukemia (AML) with myelodysplasia-related changes (AML-MRC). Therefore, despite initially being studied in the $\mathrm{r} / \mathrm{r}$ AML setting, it is not approved for relapsed AML patients per se. However, it should be considered for patients that meet the AMLMRC criteria and have failed prior HMA therapy for advanced MDS.

\section{Other non-targeted therapies}

Other therapies have emerged in first-line therapy of AML. Glasdegib, a small molecular inhibitor of the sonic hedgehog pathway, has been approved by the FDA for newly diagnosed elderly AML patients in combination with LDAC therapy. The approval was based on phase II study with elderly AML or high-risk MDS patients being randomized to the combination therapy of glasdegib and LDAC (88 patients) versus LDAC alone (44 patients). The median OS was 8.8 months with combination therapy and 4.9 months with LDAC alone [24]. Whether glasdegib also works in the r/r AML setting is currently still unknown.

\section{Novel substances—targeted}

\section{FLT3 inhibition}

FLT3-ITD and FLT3-TKD mutations are common mutations in AML with fre-

quencies of $20-25 \%$ and $6-10 \%$, respectively $[25,26]$. Importantly, they are unstable mutations that are frequently lost or gained at the time of relapse. FLT3ITD specifically is a strong adverse prognostic marker at the time of relapse and only a minority of FLT3-ITD-positive patients can be cured at the time of relapse despite aggressive salvage therapy [27]. Due to the strong independent adverse effect, FLT3ITD mutation status has been integrated into the prognostic GOELAMS score [4]. Thus, targeting FLT3 with the aim of overcoming the adverse prognostic effect is attractive. Several tyrosinkinase inhibitors have been developed with activity against FLT3 [28]. The first-generation TKIs (e.g., sorafenib, midostaurin) are multitarget 
inhibitors that function relatively unselectively against FLT3. In contrast, the nextgeneration TKIs (gilteritinib, quizartinib) have a stronger selectivity for FLT3 [28]. Due to the positive results in the RATIFY trial [29], midostaurin has been approved for FLT3-mutated AML in first-line therapy in combination with intensive chemotherapy by the FDA and EMA. It is now standard treatment for newly diagnosed FLT3-mutated AML patients in combination with intensive therapy [29]. However, midostaurin and sorafenib have little activity as monotherapy in relapsed patients [30,31]. However, next-generation TKIs have shown antileukemic single-agent activity in early clinical trials [28]. Gilteritinib is an oral type 1 FLT inhibitor that was first investigated as monotherapy in $252 \mathrm{r} / \mathrm{r}$ AML patients in a phase Ib/II trial [32]. Patients with FLT3 mutations had the best response with an ORR of 49\% compared with $12 \%$ in FLT3 wild-type patients. These promising results in FLT3mutated AML have led the way for a large randomized phase III trial in r/r FLT3mutated AML. Here, monotherapy with gilteritinib was compared with chemotherapy (ADMIRAL trial) [33••]. Chemotherapy in the control arm was performed according to the investigators choice with MEC (mitoxantrone, etoposide, and cytarabine), FLAG-IDA, LDAC, or azacitidine. Of the originally 371 patients enrolled in the trial, 247 patients received gilteritinib and 124 patients standard chemotherapy. The $\mathrm{CR}$ rate was $21.1 \%$ with gilterinib compared with 10.5 in the comparison arm. Median OS was significantly better in the gilteritinib compared with standard therapy arm (9.3 versus 5.6 months) and 1-year OS was 37.1\% versus $16.7 \%$, respectively. The EFS was 2.8 months in the gilteritinib arm and 0.7 months in the control arm. The best results were obtained for patients proceeding to transplant and continuing gilteritinib treatment thereafter. Importantly, subgroup analysis of the trial showed that a response to gilteritinib was also seen in patients with prior use of FLT3 inhibitors while patients with unfavorable cytogenetics did not seem to benefit. The results of the ADMIRAL trial were the basis for the approval of gilterinib in r/r FLT3-mutated AML by the FDA. We would recommend gilteritinib for patients with FLT3-mutated r/r AML if accessible. Quizartinib is also a next-generation TKI that is relatively selective for FLT3. It was tested in a dose-escalation phase I trial in r/r FLT3-ITD-positive AML patients [34]. Here, monotherapy with the substance yielded a CR of $37.5 \%$. In a phase III randomized trial, quizartinib monotherapy was compared with salvage chemotherapy in $\mathrm{r} / \mathrm{r}$ FLT3-ITD-positive AML [35]. Salvage chemotherapy had to be preselected and included LDAC, FLAG-IDA, or MEC. OS was significantly longer in the investigational arm versus standard arm (6.2 versus 4.7 months) with manageable toxicities. Despite the positive phase III results, the FDA and EMA have not granted approval of the substance in $\mathrm{r} / \mathrm{r}$ FLT3-ITD-positive AML patients. Crenolanib, another nextgeneration TKI with promising results in a phase II trial, is currently also studied in a phase III trial for r/r FLT3-mutated AML (NCT03250338). In summary, nextgeneration TKIs with specific inhibitory effects for mutated FLT3 are promising in $\mathrm{r} / \mathrm{r}$ AML. While midostaurin has received approval for FLT3-mutated AML in first line, gilteritinib is now approved for r/r FLT3-mutated AML by the FDA and EMA. It will be interesting to await the results of combination therapy, e.g., gilteritinib with venetoclax, in r/r FLT3-mutated AML (NCT03625505).

IDH1 and IDH2 encode for enzymes that catalyze the conversion of isocitrate to a-ketoglutarate in the cytosol and peroxisomes (IDH1) and mitochondria 
(IDH2). IDH1 and IDH2 mutations are recurrent mutations in AML with a frequency of $7-14 \%$ and $8-15 \%$, respectively $[26,36,37]$. A key pathophysiological consequence of mutant IDH1 and IDH2 is the production of the oncometablite 2-hydroxgluterate (2-HG) [38]. While IDH1/2 mutations do not have major prognostic relevance $[19,20]$, they have gained clinical significance due to the development of targeted therapies. Oral inhibitors have been introduced that inhibit the mutant form of the enzyme and block the production of 2-HG.

Ivosidenib is an oral, targeted, small-molecule inhibitor of mutant IDH1. In a phase I dose-escalation and expansion trial, 258 AML patients with IDH1

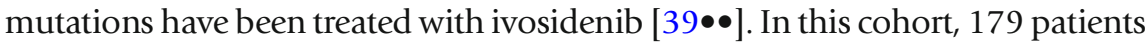
had $\mathrm{r} / \mathrm{r}$ AML. Of these $\mathrm{r} / \mathrm{r}$ patients, monotherapy with ivosidenib yielded a CR rate of $21.8 \%$. The median time to CR was 2.8 months and the median duration of CR was 9.3 months. An additional $11.7 \%$ of patients achieved a CRi or a CRp (CR with partial platelet recovery) and $38.5 \%$ of patients had stable disease. Patients with response to ivosidenib had fewer infections and episodes of febrile neutropenia. No common mutational pattern predicting response or resistance to therapy was identified. Importantly, this therapy was overall well tolerated and allows outpatient oral therapy. Common side effects included prolongation of the QT interval in $7.8 \%$ of patients and hematological toxicities in $1.7-3.4 \%$ of patients. A special side effect to IDH inhibition is the IDH differentiation syndrome (DS) which occurred in $3.9 \%$ of patients in this

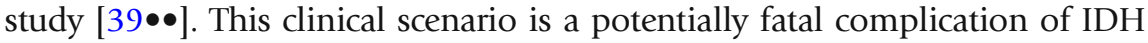
inhibitor therapy and similar to that seen in acute promyelocytic leukemia during treatment with all-trans retinoic acid and/or arsenic trioxide. Symptoms include dyspnea, unexplained fever, pulmonary infiltrates, hypoxia, and pleural effusions. These patients can show leukocytosis with signs of maturation; however, it is not mandatory. It is critical to identify IDH-DS early and treat it with corticosteroids. Similar to ivosidenib for IDH1-mutant AML, enasidenib has been developed for IDH2-mutant AML. Enasidenib is an allosteric inhibitor that binds to the IDH2 dimer interface and blocks the production of 2-HG of IDH2 mutants. In a phase I/II trial dose-escalation and expansion trial, 239 patients with IDH2-mutant advanced myeloid malignancies were treated [40, $41 \bullet \bullet$. Among this cohort, 176 patients had r/r disease. In patients with $\mathrm{r} / \mathrm{r} \mathrm{AML}$, enasidenib therapy yielded an overall response rate of $40.3 \%$. The median time to first response was 1.9 months and $87.3 \%$ of responding patients showed a first response by cycle 5 . One hundred milligrams of enasidenib daily was chosen as the dose for the expansion cohort based on plasma drug concentration, 2-HG inhibition, and clinical activity. With $100 \mathrm{mg}$ enasidenib daily, $38.5 \%$ of $\mathrm{r} / \mathrm{r}$ patients achieved a response and $20.2 \%$ of patients a CR. The duration of response ranged from 3.8 to 9.7 months (median 5.6 months). The median OS for $\mathrm{r} / \mathrm{r}$ was 9.3 months with an estimated 1-year OS of $39 \%$. Interestingly, the drug works independent of the mutational load of IDH2. A rise in indirect bilirubin serum level was observed in 35\% of patients. However, it was not a sign of intrinsic liver toxicity but instead due to off-target inhibition of the UGT1A1 enzyme. $9.6 \%$ of patients developed IDH-DS, with grade 3 or 4 in $6 \%$ of patients. The mechanisms underlying the evolving resistance to IDH inhibition after initial response have been studied. It was shown that resistance to evosidenib can emerge through the development of second-site IDH2 mutations in trans that inhibit the binding of the drug [42]. Ivosidenib and 


\section{Future directions}

enasidenib represent an important progress in the treatment of $\mathrm{r} / \mathrm{r}$ AML patients with targeted therapy. Both drugs have been approved by the FDA for $\mathrm{r} / \mathrm{r}$ AML patients with an IDH1 and IDH2 mutation respectively. Current clinical trials concentrate on combination therapies of ivosidenib or enasidenib with azacitidine (NCT03683433) or CPX-351 (NCT03825796) in r/r AML patients as well as with induction chemotherapy in newly diagnosed patients (NCT03839771).

Currently, we have little data about the efficacy and toxicity of combining novel substances with each other or with chemotherapy in $r / r$ AML. It will be important to gain prospective data in randomized trials that study venetoclax with salvage chemotherapy or other targeted therapies (e.g., gilteritinib for FLT3-mutated r/r AML or ivosidenib/ enasidenib in IDH1-/IDH2-mutated r/r AML). Cellular therapies such as CAR-T cells and bispecific antibody are less advanced in AML compared with ALL. CD33- and CD123-directed bispecific antibodies have been developed. These bispecific antibodies are also called dual-affinity retargeted antibodies. AMG 330 is anti-CD33 bispecific T cell engager antibody construct that was studied in $\mathrm{r} / \mathrm{r}$ AML patients in a phase I trial. It was a dose-escalation study that intended to find the optimal dosing schedule. Of 40 patients, the majority of patients discontinued therapy $(n=35)$ with a median of one cycle being applied. Of the 35 patients who discontinued therapy, 27 patients $(77 \%)$ discontinued due to disease progression. A total of $73 \%$ of patients experienced serious adverse events with cytokine release syndrome being very common $(28 \%$ of patients) [43]. Similar dose-finding studies are ongoing with flotetuzumab, a CD123xCD3 dual-affinity retargeted antibody. CD123 is also target of a donor-derived anti-CD123-CAR-T cell that is currently tested in relapsed AML after allo-HSCT (NCT03114670). However, finding the ideal target remains challenging both for dual-affinity retargeted antibodies as well as for CAR-T cell therapy.

$\mathrm{R} / \mathrm{R}$ AML remains a common clinical scenario with poor outcome. HSCT is necessary for long-term remission and survival. Due to the limited treatment options in relapsed patients, clinical trials are the first choice of therapy. For FLT3- and IDH1-/IDH2-mutated AML patients, targeted therapies have shown results superior to standard therapy. The FDA has approved gilteritinib for FLT3-mutated AML as well as ivosidenib/ enasidenib for IDH1-/IDH2-mutated r/r AML patients. Monotherapy with venetoclax, a bcl-2 inhibitor, has moderate efficacy in $\mathrm{r} / \mathrm{r}$ AML. However, early results in combination with intensive chemotherapy or HMA are very encouraging. Trials combining novel substances with each are necessary. The development of cellular therapies such as CAR-T cells or bispecific $\mathrm{T}$ cell engager antibody constructs is very demanding in AML as it is difficult to identify the ideal target structure on AML blasts. 


\section{Funding Information}

Open Access funding provided by Projekt DEAL.

\section{Compliance with Ethical Standards}

\section{Conflict of Interest}

Felicitas Thol has received research funding from and served on advisory boards for Novartis, Pfizer, Celgene, AbbVie, Daiichi Sankyo, Astellas, and Jazz Pharmaceuticals.

Arnold Ganser has received compensation from Novartis and Celgene for service as a consultant.

Human and Animal Rights and Informed Consent

This article does not contain any studies with human or animal subjects performed by any of the authors.

\section{Open Access}

This article is licensed under a Creative Commons Attribution 4.0 International License, which permits use, sharing, adaptation, distribution and reproduction in any medium or format, as long as you give appropriate credit to the original author(s) and the source, provide a link to the Creative Commons licence, and indicate if changes were made. The images or other third party material in this article are included in the article's Creative Commons licence, unless indicated otherwise in a credit line to the material. If material is not included in the article's Creative Commons licence and your intended use is not permitted by statutory regulation or exceeds the permitted use, you will need to obtain permission directly from the copyright holder. To view a copy of this licence, visit http://creativecommons.org/licenses/by/4.0/.

\section{References and Recommended Reading}

Papers of particular interest, published recently, have been highlighted as:

-• Of major importance

1. Dohner H, Weisdorf DJ, Bloomfield CD. Acute myeloid leukemia. N Engl J Med. 2015;373(12):1136-52.

2. Arfons LM, Tomblyn M, Rocha V, Lazarus HM. Second hematopoietic stem cell transplantation in myeloid malignancies. Curr Opin Hematol. 2009;16(2):11223.

3.• Dohner H, Estey E, Grimwade D, Amadori S, Appelbaum FR, Buchner T, et al. Diagnosis and management of AML in adults: 2017 ELN recommendations from an international expert panel. Blood. 2017;129(4):42447

Provides the present standard for the diagnosis and management of relapsed and refractory AML.

4. Chevallier P, Labopin M, Turlure P, Prebet T, Pigneux A, Hunault $\mathrm{M}$, et al. A new leukemia prognostic scoring system for refractory/relapsed adult acute myelogeneous leukaemia patients: a GOELAMS study. Leukemia. 2011;25(6):939-44.
5. Thol F, Schlenk RF, Heuser M, Ganser A. How I treat refractory and early relapsed acute myeloid leukemia. Blood. 2015;126(3):319-27.

6. Pastore D, Specchia G, Carluccio P, Liso A, Mestice A, Rizzi R, et al. FLAG-IDA in the treatment of refractory/ relapsed acute myeloid leukemia: single-center experience. Ann Hematol. 2003;82(4):231-5.

7. Breems DA, Van Putten WL, Huijgens PC, Ossenkoppele GJ, Verhoef GE, Verdonck LF, et al. Prognostic index for adult patients with acute myeloid leukemia in first relapse. J Clin Oncol. 2005;23(9):1969-78.

8. Bejanyan N, Weisdorf DJ, Logan BR, Wang HL, Devine SM, de Lima M, et al. Survival of patients with acute myeloid leukemia relapsing after allogeneic hematopoietic cell transplantation: a center for international blood and marrow transplant research study. Biol Blood Marrow Transplant. 2015;21(3):454-9.

9. Kharfan-Dabaja MA, Labopin M, Polge E, Nishihori T, Bazarbachi A, Finke J, et al. Association of second 
allogeneic hematopoietic cell transplant vs donor lymphocyte infusion with overall survival in patients with acute myeloid leukemia relapse. JAMA Oncol. 2018;4(9):1245-53.

10. Dombret H, Seymour JF, Butrym A, Wierzbowska A Selleslag D, Jang JH, et al. International phase 3 study of azacitidine vs conventional care regimens in older patients with newly diagnosed AML with $>30 \%$ blasts. Blood. 2015;126(3):291-9.

11. Vo TT, Ryan J, Carrasco R, Neuberg D, Rossi DJ, Stone $\mathrm{RM}$, et al. Relative mitochondrial priming of myeloblasts and normal HSCs determines chemotherapeutic success in AML. Cell. 2012;151(2):344-55.

12. Pan R, Hogdal LJ, Benito JM, Bucci D, Han L, Borthakur G, et al. Selective BCL-2 inhibition by ABT-199 causes on-target cell death in acute myeloid leukemia. Cancer Discov. 2014;4(3):362-75.

13. Konopleva M, Pollyea DA, Potluri J, Chyla B, Hogdal L, Busman T, et al. Efficacy and biological correlates of response in a phase II study of venetoclax monotherapy in patients with acute myelogenous leukemia. Cancer Discov. 2016;6(10):1106-17.

14. Huemer F, Melchardt T, Jansko B, Wahida A, Jilg S, Jost $\mathrm{PJ}$, et al. Durable remissions with venetoclax monotherapy in secondary AML refractory to hypomethylating agents and high expression of BCL-2 and/or BIM. Eur J Haematol. 2019;102(5):437-41.

15. DiNardo CD, Pratz K, Pullarkat V, Jonas BA, Arellano M, Becker PS, et al. Venetoclax combined with decitabine or azacitidine in treatment-naive, elderly patients with acute myeloid leukemia. Blood. 2019;133(1):7-17.

16. Wei AH, Strickland SA Jr, Hou JZ, Fiedler W, Lin TL, Walter RB, et al. Venetoclax combined with low-dose cytarabine for previously untreated patients with acute myeloid leukemia: results from a phase Ib/II study. J Clin Oncol. 2019;37(15):1277-84.

17. Ram R, Amit O, Zuckerman T, Gurion R, Raanani $P$, Bar-On Y, et al. Venetoclax in patients with acute myeloid leukemia refractory to hypomethylating agents-a multicenter historical prospective study. Ann Hematol. 2019;98(8):1927-32.

18. Aldoss I, Yang D, Aribi A, Ali H, Sandhu K, Al Malki $\mathrm{MM}$, et al. Efficacy of the combination of venetoclax and hypomethylating agents in relapsed/refractory acute myeloid leukemia. Haematologica. 2018;103(9):e404-e7.

19. Shahswar R, Beutel G, Klement P, Rehberg A, Gabdoulline R, Koeneck C, et al. FLA-IDA salvage chemotherapy combined with a seven-day course of venetoclax (FLAVIDA) in patients with relapsed/refractory acute leukemia. Br J Haematol. 2019;in Press.

20. DiNardo CD, Albitar M, Kadia TM, Naqvi K, Vaughan $\mathrm{K}$, Cavazos A. Venetoclax in combination with FLAGIDA chemotherapy (FLAG-V-I) for fit, relapsed/ refractory AML patients: interim results of a phase $1 \mathrm{~b} / 2$ dose escalation and expansion study. ASH Abstract. 2018;4048.

21. Feldman EJ, Lancet JE, Kolitz JE, Ritchie EK, Roboz GJ, List AF, et al. First-in-man study of CPX-351: a liposomal carrier containing cytarabine and daunorubicin in a fixed 5:1 molar ratio for the treatment of relapsed and refractory acute myeloid leukemia. J Clin Oncol. 2011;29(8):979-85.

22. Cortes JE, Goldberg SL, Feldman EJ, Rizzeri DA, Hogge DE, Larson $\mathrm{M}$, et al. Phase II, multicenter, randomized trial of CPX-351 (cytarabine:daunorubicin) liposome injection versus intensive salvage therapy in adults with first relapse AML. Cancer. 2015;121(2):234-42.

23. Lancet JE, Uy GL, Cortes JE, Newell LF, Lin TL, Ritchie EK, et al. CPX-351 (cytarabine and daunorubicin) liposome for injection versus conventional cytarabine plus daunorubicin in older patients with newly diagnosed secondary acute myeloid leukemia. J Clin Oncol. 2018;36(26):2684-92.

24. Cortes JE, Heidel FH, Hellmann A, Fiedler W, Smith $\mathrm{BD}$, Robak T, et al. Randomized comparison of low dose cytarabine with or without glasdegib in patients with newly diagnosed acute myeloid leukemia or highrisk myelodysplastic syndrome. Leukemia. 2019;33(2):379-89.

25. Network TCGAR. Genomic and epigenomic landscapes of adult de novo acute myeloid leukemia. N Engl J Med. 2013;368(22):2059-74.

26. Papaemmanuil E, Gerstung M, Bullinger L, Gaidzik VI, Paschka P, Roberts ND, et al. Genomic classification and prognosis in acute myeloid leukemia. $\mathrm{N}$ Engl J Med. 2016;374(23):2209-21.

27. Wagner K, Damm F, Thol F, Gohring G, Gorlich $\mathrm{K}$, Heuser $\mathrm{M}$, et al. FLT3-internal tandem duplication and age are the major prognostic factors in patients with relapsed acute myeloid leukemia with normal karyotype. Haematologica. 2011;96(5):681-6.

28. Daver N, Schlenk RF, Russell NH, Levis MJ. Targeting FLT3 mutations in AML: review of current knowledge and evidence. Leukemia. 2019;33(2):299-312.

29. Stone RM, Mandrekar SJ, Sanford BL, Laumann K, Geyer S, Bloomfield CD, et al. Midostaurin plus chemotherapy for acute myeloid leukemia with a FLT3 mutation. N Engl J Med. 2017;377(5):454-64.

30. Fischer T, Stone RM, Deangelo DJ, Galinsky I, Estey E, Lanza C, et al. Phase IIB trial of oral midostaurin (PKC412), the FMS-like tyrosine kinase 3 receptor (FLT3) and multi-targeted kinase inhibitor, in patients with acute myeloid leukemia and high-risk myelodysplastic syndrome with either wild-type or mutated FLT3. J Clin Oncol. 2010;28(28):4339-45.

31. Stone RM, DeAngelo DJ, Klimek V, Galinsky I, Estey E, Nimer SD, et al. Patients with acute myeloid leukemia and an activating mutation in FLT3 respond to a smallmolecule FLT3 tyrosine kinase inhibitor, PKC412. Blood. 2005;105(1):54-60.

32. Perl AE, Altman JK, Cortes J, Smith C, Litzow M, Baer $\mathrm{MR}$, et al. Selective inhibition of FLT3 by gilteritinib in relapsed or refractory acute myeloid leukaemia: a multicentre, first-in-human, open-label, phase 1-2 study. Lancet Oncol. 2017;18(8):1061-75. 
33.•Perl AE, Martinelli G, Cortes JE, Neubauer A, Berman E, Paolini S, et al. Gilteritinib or Chemotherapy for relapsed or refractory FLT3-mutated aml. N Engl J Med. 2019;381(18):1728-40

Demonstrates the superiority of gilteritinib over chemotherapy in $\mathrm{r} / \mathrm{r}$ FLT3-mutated AML.

34. Usuki $\mathrm{K}$, Handa H, Choi I, Yamauchi T, Iida H, Hata T, et al. Safety and pharmacokinetics of quizartinib in Japanese patients with relapsed or refractory acute myeloid leukemia in a phase 1 study. Int J Hematol. 2019;110(6):654-64.

35. Cortes JE, Khaled S, Martinelli G, Perl AE, Ganguly S, Russell N, et al. Quizartinib versus salvage chemotherapy in relapsed or refractory FLT3-ITD acute myeloid leukaemia (QuANTUM-R): a multicentre, randomised, controlled, open-label, phase 3 trial. Lancet Oncol. 2019;20(7):984-97.

36. Thol F, Damm F, Wagner K, Gohring G, Schlegelberger B, Hoelzer D, et al. Prognostic impact of IDH2 mutations in cytogenetically normal acute myeloid leukemia. Blood. 2010;116(4):614-6.

37. Wagner K, Damm F, Gohring G, Gorlich K, Heuser M, Schafer I, et al. Impact of IDH1 R132 mutations and an IDH1 single nucleotide polymorphism in cytogenetically normal acute myeloid leukemia: SNP rs11554137 is an adverse prognostic factor. J Clin Oncol. 2010;28(14):2356-64.

38. Dang L, White DW, Gross S, Bennett BD, Bittinger MA Driggers EM, et al. Cancer-associated IDH1 mutations produce 2-hydroxyglutarate. Nature. 2009;462(7274):739-44.

$39 . \bullet$ DiNardo CD, Stein EM, de Botton S, Roboz GJ, Altman JK, Mims AS, et al. Durable remissions with ivosidenib in IDH1-mutated relapsed or refractory AML. N Engl J
Med. 2018;378(25):2386-98

Provides the clinical data leading to FDA approval of ivosidenib for the treatment of IDH1-mutated r/rAML.

40. Stein EM, DiNardo CD, Fathi AT, Pollyea DA, Stone RM, Altman JK, et al. Molecular remission and response patterns in patients with mutant-IDH2 acute myeloid leukemia treated with enasidenib. Blood. 2019;133(7):676-87.

41.• Stein EM, DiNardo CD, Pollyea DA, Fathi AT, Roboz GJ, Altman JK, et al. Enasidenib in mutant IDH2 relapsed or refractory acute myeloid leukemia. Blood. 2017;130(6):722-31

Provides the clinical data leading to FDA approval of enasidenib for the treatment of IDH2-mutated r/rAML.

42. Intlekofer AM, Shih AH, Wang B, Nazir A, Rustenburg AS, Albanese SK, et al. Acquired resistance to IDH inhibition through trans or cis dimer-interface mutations. Nature. 2018;559(7712):125-9.

43. Ravandi F, Stein AS, Kantarjian HM, Walter RB, Paschka, Jongen-Lavrencic $M$, et al. A phase 1 first-inhuman study of AMG 330, an Anti-CD33 bispecific Tcell engager $\left(\mathrm{BiTE}^{\circledR}\right)$ antibody construct, in relapsed/ refractory acute myeloid leukemia (R/R AML). ASH Annual Meeting. 2018;\# 25.

\section{Publisher's note}

Springer Nature remains neutral with regard to jurisdictional claims in published maps and institutional affiliations. 